\title{
CONJUGACY CLASSES OF LEFT IDEALS OF A FINITE DIMENSIONAL ALGEBRA
}

\author{
Arkadiusz MȨCEL AND JAN OKNIŃSKI
}

\begin{abstract}
Let $A$ be a finite dimensional unital algebra over a field $K$ and let $C(A)$ denote the set of conjugacy classes of left ideals in $A$. It is shown that $C(A)$ is finite if and only if the number of conjugacy classes of nilpotent left ideals in $A$ is finite. The set $C(A)$ can be considered as a semigroup under the natural operation induced from the multiplication in $A$. If $K$ is algebraically closed, the square of the radical of $A$ is zero and $C(A)$ is finite, then for every $K$-algebra $B$ such that $C(B) \cong C(A)$ it is shown that $B \cong A$.
\end{abstract}

2010 Mathematics Subject Classification: 16P10, 16D99, 20M99.

Key words: Finite dimensional algebra, left ideal, semigroup, conjugacy class.

\section{Introduction}

Throughout the paper $A$ is a finite dimensional algebra with unity over a field $K$. Let $U(A)$ be the group of units of $A$. By $L(A)$ we denote the set of left ideals of $A$. An equivalence relation $\sim$ can be introduced on $L(A)$ by identifying $L_{1}, L_{2} \in L(A)$ such that $L_{1}=L_{2} u$, for some $u \in U(A)$. The set of equivalence classes $L(A) / \sim$, denoted by $C(A)$, clearly coincides with the set of conjugacy classes of left ideals in $A$. It is easy to see that $C(A)$ admits a semigroup structure defined by: $\left[L_{1}\right]\left[L_{2}\right]=\left[L_{1} L_{2}\right]$ for $L_{1}, L_{2} \in L(A)$.

The definition of $C(A)$ was introduced in [8]. The finiteness of $C(A)$ was proved to be strongly related to the finite representation type property of finite dimensional algebras. Recall that an algebra $A$ is of finite representation type if and only if there are finitely many isomorphism classes of indecomposable left $A$-modules of finite length, see [2]. Namely, if $A$ is of finite type then $C(A)$ is finite. On the other hand, if the field $K$ is infinite and all semigroups $C\left(M_{n}(A)\right)$, associated to matrix algebras $M_{n}(A)$, for $n \geq 1$, are finite then $A$ is of finite type, see Theorem 6 and Theorem 7 in [8].

Research partially supported by MNiSW research grant N201 420539 . 
Another motivation for a study of finiteness of the semigroup $C(A)$ follows from its relation to the double cosets $U(A) a U(A)$ for $a \in A$. Namely, if $C(A)$ is finite then the number of such cosets also is finite. The latter condition is equivalent to the distributivity of the lattice of (two-sided) ideals of $A$, provided that $K$ is infinite.

A complete characterization of algebras for which the semigroup of conjugacy classes of left ideals is finite has been obtained for the class of basic algebras over an algebraically closed field with 2-nilpotent Jacobson radical (see $[\mathbf{8}$, Theorem 12$]$ ). Recall that an algebra $\mathrm{A}$ with a complete set $\left\{e_{1}, e_{2}, \ldots, e_{n}\right\}$ of primitive orthogonal idempotents is called basic if $A e_{i} \neq A e_{j}$ (as left $A$-modules), for all $i \neq j$. We note that the structure of rings $R$ with a finite number of cosets $U(R) a, a \in R$, was completely determined in $[\mathbf{5}]$.

The aim of the present paper is to continue the investigation of $C(A)$. The main idea is to look for finite invariants of an algebra $A$ that can be expressed in terms of $C(A)$. The two major motivating problems can be formulated as follows:

Problem 1. Determine necessary and sufficient conditions under which $C(A)$ is finite.

Problem 2. Determine properties of the algebra $A$ that can be recognized by the semigroup $C(A)$. In particular, does $C(A) \cong C(B)$ for a $K$-algebra $B$ imply that $A \cong B$, provided that the field $K$ is algebraically closed?

Note that, for any division algebra $D$, the semigroup $C\left(M_{n}(D)\right)$ is isomorphic to the semigroup with zero $\left\{e_{1}, \ldots, e_{n}\right\} \cup\{0\}$, where the operation is defined by: $e_{i} e_{j}=e_{j}$, for $i, j=1, \ldots, n$. Hence, the hypothesis on the field $K$ is necessary.

Our first main result contributes to the former problem.

Theorem 1.1. Let $A$ be a finite dimensional algebra over an arbitrary field $K$. The following conditions are equivalent:

1) $C(A)$ is finite,

2) the number of conjugacy classes of nilpotent left ideals in $A$ is finite.

In the context of representation theory of finite dimensional algebras, and in view of the above example, it is natural to work under the hypothesis that the base field $K$ is algebraically closed. Moreover, several proofs are based on the important standard reductions to the class of basic algebras or/and to the case where the radical $J(A)$ of $A$ is 2-nilpotent, see $[\mathbf{2}]$ and $[\mathbf{9}]$. The second main result of this paper is a contribution to Problem 2. 
Theorem 1.2. Let $A, B$ be finite dimensional algebras over an algebraically closed field $K$. Assume that $J(A)^{2}=0$ and $C(A)$ is finite. If the semigroups $C(A)$ and $C(B)$ are isomorphic then the algebras $A$ and $B$ are isomorphic.

The material is divided into five sections. In Section 2 we recall the necessary prerequisites on idempotents and some semigroup-theoretical tools used in the paper. The proof of Theorem 1.1 is given in Section 3. Some comments on the connections between the case of arbitrary algebras and the case of basic algebras are given in Section 4. A discussion of the properties of $A$ that can be recognized from the structure of $C(A)$ is started in Section 5. Then the proof of Theorem 1.2 follows in Section 6 .

\section{Prerequisites}

Throughout the paper $K$ denotes an infinite field and $A$ denotes a finite dimensional algebra over $K$. By $J(A)$ we mean the Jacobson radical of $A$. The image of $a \in A$ under the natural map $\pi: A \rightarrow A / J(A)$ is denoted by $\bar{a}$. We write $\bar{A}=A / J(A)$. We begin with some known facts concerning the conjugacy classes of idempotents in $A$ and the principal left ideals generated by such elements.

Lemma 2.1. The number of conjugacy classes of idempotents in $A$ is finite.

Proof: It is well known that every two decompositions of unity into a sum of primitive orthogonal idempotents are conjugate, see Theorem 3.4.1 in [4]. Hence the assertion follows easily.

Proposition 2.2. Let $e, f$ be idempotents in A. The following conditions are equivalent:

(i) $\bar{A} \bar{e} \cong \overline{A f}$ as left $\bar{A}$-modules,

(ii) $A e \cong A f$ as left $A$-modules,

(iii) $\bar{e}$ and $\bar{f}$ are conjugate in $\bar{A}$,

(iv) $e$ and $f$ are conjugate in $A$,

(v) $[\bar{A} \bar{e}]=[\overline{A f}]$ in $C(\bar{A})$,

(vi) $[A e]=[A f]$ in $C(A)$,

(vii) $e$ and $f$ generate the same ideal in the multiplicative monoid $(A, \cdot)$. 
Proof: For the sake of completeness, we sketch the proof. It is enough to check that

$$
\begin{gathered}
(\mathrm{i}) \Rightarrow(\mathrm{ii}) \Rightarrow(\mathrm{iv}) \Rightarrow(\text { iii }) \Rightarrow(\mathrm{v}) \Rightarrow(\mathrm{i}) \\
(\mathrm{iv}) \Rightarrow(\mathrm{vi}) \Rightarrow(\mathrm{ii}) \text { and }(\mathrm{iv}) \Rightarrow(\text { vii }) \Rightarrow(\mathrm{ii}) .
\end{gathered}
$$

Only the implications (i) $\Rightarrow$ (ii), (ii) $\Rightarrow$ (iv) and (vii) $\Rightarrow$ (ii) require a proof.

To deduce (ii) from (i) observe that $\bar{A} \bar{e} \cong A e / J(A) e$ and an isomorphism between $\bar{A} \bar{e}$ and $\overline{A f}$ can be lifted to an isomorphism between $A e$ and $A f$ (see Proposition 17.18 in [1]).

Suppose that $A e \cong A f$ as $A$-modules. It is known that

$$
\operatorname{Hom}(A e, A f) \cong e A f,
$$

and every homomorphism $\phi: A e \rightarrow A f$ can be described by the formula $\phi(x)=x(e a f)$, for $x \in A e$ where $a \in A$ (see Corollary 6.4b in [9]). Assume that $\phi$ is an isomorphism. There exists $f b e \in f A e$ such that $\phi^{-1}(y)=y(f b e)$. Hence we have

$$
e=e a f \cdot f b e, \quad f=f b e \cdot e a f,
$$

for some $a, b \in A$. In view of the following decompositions of left $A$-modules

$$
A=A e \oplus A(1-e), \quad A=A f \oplus A(1-f)
$$

from Krull-Schmidt theorem it follows that $A(1-e) \cong A(1-f)$. Hence, as above we get

$$
\begin{aligned}
& 1-f=(1-f) c(1-e) \cdot(1-e) d(1-f) \\
& 1-e=(1-e) d(1-f) \cdot(1-f) c(1-e)
\end{aligned}
$$

for some $c, d \in A$. Put

$$
u=e a f+(1-e) d(1-f), \quad v=f b e+(1-f) c(1-e) .
$$

According to (2) and (3) we have $u v=1$ and $e u=u f$. Thus (ii) $\Rightarrow$ (iv).

Assume that (vii) holds. From Theorem 2.20 in [3] it follows that there exist $a, b \in A$ such that $e a f$ and $f b e$ belong to the ideal of the semigroup $(A, \cdot)$ generated by $e$ and such that $(f b e)(e a f)=f,(e a f)(f b e)=$ $e$. In view of (1) these two elements yield an isomorphism $A e \cong A f$. Hence (ii) follows.

Let us note that condition (vii) does not usually appear in ringtheoretic considerations. On the other hand, it plays an important role in the theory of connected algebraic monoids, see Corollary 6.8 in [10]. We shall see that it is very useful also in the setting of finite dimensional 
algebras (which are connected monoids if the field $K$ is algebraically closed).

Although Theorem 1.1 is concerned with ring-theoretic properties of the algebra $A$, its proof depends heavily on certain semigroup-theoretic considerations. For standard facts on semigroups that are used in the paper we refer the reader to $[\mathbf{3}]$. In particular, we use Green relations $\mathcal{R}$, $\mathcal{L}, \mathcal{H}, \mathcal{J}$ defined on any semigroup $S$ and the so called egg-box pattern on completely 0 -simple semigroups.

Definition 2.3. We say that $H$ is a subgroup of a semigroup $S$ if $H$ is a subgroup of the unit group $U(e S e)$ of the monoid $e S e$, for some idempotent $e \in S$.

A semigroup $S$ is called strongly $\pi$-regular if for every $s \in S$ there exists $n \geq 1$ such that $s^{n}$ is contained in a subgroup of $S$.

We will need the following consequence of the well known fact that a ring $R$ has the property that for every $x \in R$ the chain $x R \supseteq x^{2} R \supseteq$ $x^{3} R \supseteq \cdots$ stabilizes if and only if the multiplicative semigroup of $R$ is strongly $\pi$-regular; for example, this easily follows from [7, Exercise 23.5 and Exercise 23.6] (for another generalization of Proposition 2.4 see also [6, Proposition 1.2]).

Proposition 2.4. Let $A$ be a finite dimensional algebra with unity. Then the multiplicative semigroup $(A, \cdot)$ of $A$ is strongly $\pi$-regular.

\section{Conjugacy classes of nilpotent ideals}

Recall the standard partial order $\leq$ on the set of all idempotents of $A$. If $e=e^{2}, f=f^{2} \in A$ then we write $e \leq f$ if $e f=f e=e$.

Lemma 3.1. Let $L \in L(A)$. The following conditions are equivalent for an idempotent $e \in L$ :

(i) $e$ is a maximal idempotent in $L$,

(ii) the left ideal $L(1-e)$ is contained in $J(A)$.

Proof: First note that $L$ can be decomposed into a direct sum of left ideals:

$$
L=A e \oplus L(1-e) .
$$

Assume first that $L(1-e) \nsubseteq J(A)$. Then there exists a nonzero $f=$ $f^{2} \in L(1-e)$ and (4) applied to $L(1-e)$ implies that

$$
L=A e \oplus A f \oplus L(1-e)(1-f)=A e \oplus A f \oplus L(1-e-f+e f) .
$$

Observe that $f=f(1-e)$ and $f e=0$. This easily implies that

$$
(e+f-e f)^{2}=e+f-e f .
$$


Clearly

$$
e(e+f-e f)=e=(e+f-e f) e .
$$

In other words, $e \leq e+f-e f$. If $e$ is maximal in $L$, we deduce that $e=e+f-e f$. Thus $f=e f$ and $f=f^{2}=f(e f)=0$, a contradiction. This shows that (ii) is a consequence of (i).

Assume now that $L(1-e) \subseteq J(A)$. Suppose that $f \in L$ is an idempotent such that $e f=f e=e$. Clearly $f-e \in L$ and $(f-e) e=0$ so $f-e \in L(1-e)$. Since $(f-e)^{2}=f-e$, it follows that $f-e=0$. Thus (ii) $\Rightarrow$ (i).

Corollary 3.2. Let $e, e^{\prime}$ be maximal idempotents in a left ideal $L$ of $A$. Then $e, e^{\prime}$ are conjugate in $A$.

Proof: In view of Lemma 3.1 and (4) we get that $\bar{A} \bar{e}=\overline{A e^{\prime}}$. Hence, the assertion follows from Proposition 2.2.

Proposition 3.3. Let $[I],[J] \in C(A)$. The following conditions are equivalent:

(i) $[I]=[J]$,

(ii) there exist idempotents e, $e^{\prime}$ maximal in $I, J$, respectively, such that $[A e]=\left[A e^{\prime}\right]$ and $[I(1-e)]=\left[J\left(1-e^{\prime}\right)\right]$.

Proof: Assume that $[I]=[J]$ and let $e$ be a maximal idempotent in $I$. Then $J=g^{-1} I g$ for some $g \in U(A)$ and $e^{\prime}=g^{-1} e g$ is an idempotent in $J$ such that

$J\left(1-e^{\prime}\right)=J\left(1-g^{-1} e g\right)=J g^{-1}(1-e) g=g^{-1} I g g^{-1}(1-e) g=g^{-1} I(1-e) g$.

Hence $[I(1-e)]=\left[J\left(1-e^{\prime}\right)\right]$. Moreover, $e^{\prime}$ is a maximal idempotent in $J$. Therefore (ii) is a consequence of (i).

It remains to prove the implication (ii) $\Rightarrow$ (i). By Proposition 2.2 the condition $[A e]=\left[A e^{\prime}\right]$ implies that $e, e^{\prime}$ are conjugate. Hence, conjugating $I$ or $J$ we may assume that $e=e^{\prime}$, so that

$$
I=A e \oplus I(1-e), \quad J=A e \oplus J(1-e),
$$

and $I(1-e), J(1-e) \subseteq J(A)$. By the hypothesis, there exists also $g \in U(A)$ such that

$$
I(1-e)=J(1-e) g
$$

The element $g$ may be represented as a matrix of the form $g=\left(\begin{array}{ll}g_{1} & g_{2} \\ g_{3} & g_{4}\end{array}\right)$, where

$$
g_{1}=e g e, \quad g_{2}=e g(1-e), \quad g_{3}=(1-e) g e, \quad g_{4}=(1-e) g(1-e) .
$$


Let

$$
\begin{aligned}
& I_{1}=e I e, \quad I_{2}=e I(1-e), \quad I_{3}=(1-e) I e, \quad I_{4}=(1-e) I(1-e) \\
& J_{1}=e J e, \quad J_{2}=e J(1-e), \quad J_{3}=(1-e) J e, \quad J_{4}=(1-e) J(1-e) .
\end{aligned}
$$

Using matrix notation and applying (6) we obtain

$$
\left(\begin{array}{ll}
0 & I_{2} \\
0 & I_{4}
\end{array}\right)=\left(\begin{array}{ll}
0 & J_{2} \\
0 & J_{4}
\end{array}\right) \cdot\left(\begin{array}{ll}
g_{1} & g_{2} \\
g_{3} & g_{4}
\end{array}\right)=\left(\begin{array}{ll}
J_{2} g_{3} & J_{2} g_{4} \\
J_{4} g_{3} & J_{4} g_{4}
\end{array}\right) .
$$

It is also clear that there exists $h=\left(\begin{array}{ll}h_{1} & h_{2} \\ h_{3} & h_{4}\end{array}\right) \in U(A)$ such that

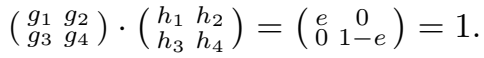

Hence

$$
g_{3} h_{2}+g_{4} h_{4}=1-e .
$$

Therefore

$$
J(1-e)=J(1-e)\left(g_{3} h_{2}+g_{4} h_{4}\right) .
$$

From $(7)$ we deduce that $J(1-e) g_{3}=\left(J_{2}+J_{4}\right) g_{3}=0$ and $J(1-e) g_{4}=$ $I(1-e)$. Thus

$$
J(1-e)=J(1-e) g_{4} h_{4}=I(1-e) h_{4} .
$$

One then easily checks that for every $n \in \mathbb{N}$ the following equalities hold

$$
\left\{\begin{array}{l}
J(1-e)\left(g_{4} h_{4}\right)^{n}=J(1-e) \\
I(1-e)\left(h_{4} g_{4}\right)^{n}=I(1-e)
\end{array} .\right.
$$

According to Proposition 2.4, the multiplicative semigroup of $A$ is strongly $\pi$-regular. Hence $\left(g_{4} h_{4}\right)^{n} \in H,\left(h_{4} g_{4}\right)^{n} \in H^{\prime}$, for some $n \in \mathbb{N}$, where $H, H^{\prime}$ are maximal subgroups of the monoid $((1-e) A(1-e), \cdot)$. Put

$$
s=\left(g_{4} h_{4}\right)^{n} g_{4} \in(1-e) A(1-e) .
$$

Observe that $s h_{4}\left(g_{4} h_{4}\right)^{n-1} \in H$, so $\left(g_{4} h_{4}\right)^{n}$ and $s$ are in the same $\mathcal{R}$-class of the multiplicative monoid $(1-e) A(1-e)$. In the same way we can see that $s$ and $\left(h_{4} g_{4}\right)^{n}$ are in the same $\mathcal{L}$-class of $(1-e) A(1-e)$. In particular, $\left(g_{4} h_{4}\right)^{n},\left(h_{4} g_{4}\right)^{n}$ are in the same $\mathcal{J}$-class of the monoid $(1-e) A(1-e)$.

Let $f, f^{\prime}$ be the unities of $H$ and $H^{\prime}$, respectively. Then $\left(g_{4} h_{4}\right)^{n} \mathcal{H} f$ and $\left(h_{4} g_{4}\right)^{n} \mathcal{H} f^{\prime}$. Since the maximal subgroups of every semigroup $S$ are precisely the $\mathcal{H}$-classes of $S$ containing idempotents (see [3, Exercise 1, $\S 2.3]$ ), the egg-box pattern (see $[3$, p. 48]) on the $\mathcal{J}$-class containing $f$ is of the following form 


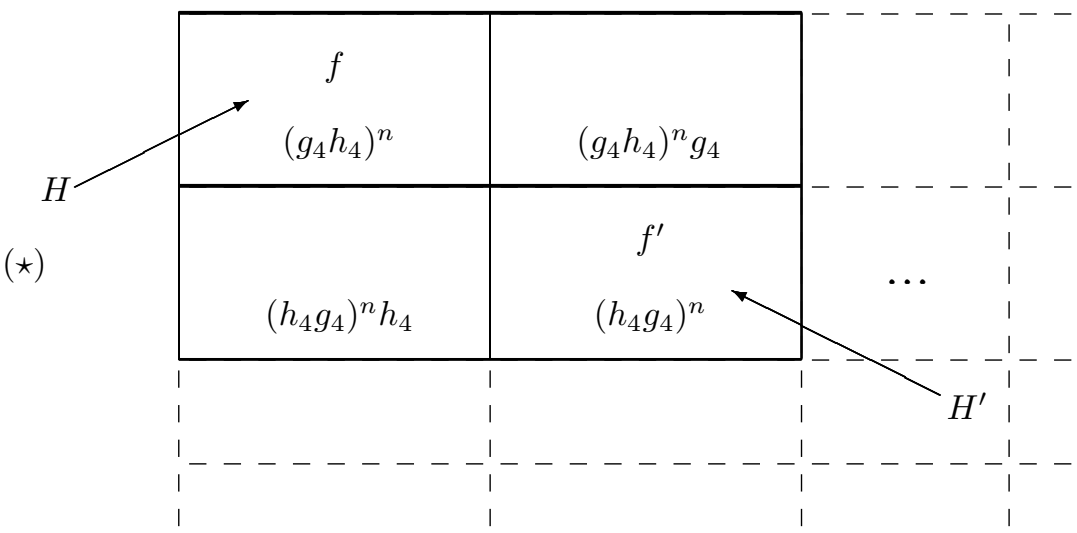

(Note that if $f, f^{\prime}$ not only lie in the same $\mathcal{J}$-class of $((1-e) A(1-e), \cdot)$, but also in the same $\mathcal{R}$ - or $\mathcal{L}$-class of this monoid, then the egg-box pattern on their $\mathcal{J}$-class becomes simpler.)

From (9), and since $f, f^{\prime} \in(1-e) A(1-e)$, we deduce that

$$
\left\{\begin{array}{l}
J(1-e)=J(1-e) f=J f \\
I(1-e)=I(1-e) f^{\prime}=I f^{\prime}
\end{array} .\right.
$$

Implication (vii) $\Rightarrow$ (iv) of Proposition 2.2 allows us to find an element $u \in U((1-e) A(1-e))$ such that in $(1-e) A(1-e)$ we have $u^{-1} f u=f^{\prime}$. Consider $\widehat{u}=e+u$. Clearly $\widehat{u} \in U(A)$ and $\widehat{u}^{-1} f \widehat{u}=f^{\prime}$. Hence, by (5) and (10) we get

$$
J \widehat{u}=(A e \oplus J f) \widehat{u}=A e \widehat{u} \oplus J f \widehat{u}=A e \oplus J \widehat{u} \widehat{u}^{-1} f \widehat{u}=A e \oplus J \widehat{u} f^{\prime} .
$$

Thus, replacing $J$ by its conjugate $J \widehat{u}$, we may assume that

$$
\left\{\begin{array}{l}
J=A e \oplus J f^{\prime} \\
I=A e \oplus I f^{\prime}
\end{array} .\right.
$$

We are, therefore, allowed to assume that $f=f^{\prime}$ and

$$
\left(g_{4} h_{4}\right)^{n},\left(h_{4} g_{4}\right)^{n} \in H=H^{\prime}=U(f A f) .
$$

Then, according to $(\star)$, also $\left(g_{4} h_{4}\right)^{n} g_{4},\left(h_{4} g_{4}\right)^{n} h_{4} \in H$. Put

$$
z=1-f+\left(g_{4} h_{4}\right)^{n} g_{4}
$$


Since $\left(g_{4} h_{4}\right)^{n} g_{4} \in U(f A f)$, we get $z \in U(A)$. Clearly $e f=0$ and it follows that

$$
\begin{aligned}
J z & =(A e \oplus J f) z=A e \oplus J f z \\
& \stackrel{(11)}{=} A e \oplus J(1-e)\left(g_{4} h_{4}\right)^{n} g_{4} \\
& \stackrel{(9)}{=} A e \oplus J(1-e) g_{4} \\
& \stackrel{(8)}{=} A e \oplus I(1-e) h_{4} g_{4} \\
& \stackrel{(9)}{=} A e \oplus I(1-e) \\
& \stackrel{(5)}{=} I .
\end{aligned}
$$

Thus $[I]=[J]$ in $C(A)$. This completes the proof of the proposition.

Proof of Theorem 1.1: Clearly, condition 2) is a consequence of 1). Thus, assume that there are finitely many conjugacy classes of nilpotent left ideals in $A$. Consider $I, J \in L(A)$. According to Lemma 3.1 there exist idempotents $e \in I$ and $e^{\prime} \in J$, maximal (respectively in $I$ and $J$ ) such that the following equalities hold

$$
I=A e \oplus I(1-e), \quad J=A e^{\prime} \oplus J\left(1-e^{\prime}\right),
$$

and $I(1-e), J\left(1-e^{\prime}\right) \subseteq J(A)$. By Lemma 2.1 we may assume that $e$ and $e^{\prime}$ are conjugate. Hence, conjugating $I$ or $J$ we may assume that $e=e^{\prime}$, so that

$$
I=A e \oplus I(1-e), \quad J=A e \oplus J(1-e),
$$

and $I(1-e), J(1-e) \subseteq J(A)$.

Since there are only finitely many conjugacy classes of left ideals in $J(A)$, we may also assume that $I(1-e)$ and $J(1-e)$ are conjugate. Therefore, Proposition 3.3 implies that $C(A)$ is finite.

Actually, the following is a direct consequence of the above proof.

Corollary 3.4. Assume that $A$ is a finite dimensional algebra with unity. Assume that $A$ has $n$ conjugacy classes of idempotents and that the number of conjugacy classes of nilpotent left ideals in $A$ is finite and equal to $m$. Then the semigroup $C(A)$ is finite and $|C(A)| \leq n m$.

\section{Basic versus non-basic algebras}

One of the motivations for the study of the semigroup $C(A)$ is to look for new (semigroup-theoretic) invariants of finite dimensional algebras. 
Having in mind the example mentioned after Problem 2 in the introduction, throughout the remainder of the paper we assume the field $K$ to be algebraically closed.

Definition 4.1. Let $A$ be a finite dimensional algebra and let $\left\{e_{1}, \ldots, e_{n}\right\}$ be a complete set of orthogonal primitive idempotents of $A$. Recall that a directed graph $\Gamma=(V, E)$ is called the quiver of $A$, which we denote by $\Gamma(A)$, if the vertex set of $\Gamma$ is of the form $V=\{1,2, \ldots, n\}$ and the edge set $E$ is equal to $\left\{(i, j) \mid e_{i} J(A) e_{j} \neq 0\right\}$ (see $[\mathbf{9}, \S 6.4]$ ). Associated with $\Gamma$ is the quiver $\Gamma^{s}=\left(V^{s}, E^{s}\right)$, where $V^{s}=V \times\{0,1\}$ and $E^{s}=\{((i, 0),(j, 1)) \mid(i, j) \in E\}$. It is called the separated quiver of $A$ and it is also denoted by $\Gamma^{s}(A)$.

The following result was proved in [8, Theorem 12].

Theorem 4.2. Let $A$ be a finite dimensional basic algebra with a distributive lattice of ideals over an algebraically closed field $K$ and such that $J(A)^{2}=0$. Then the following conditions are equivalent:

1) $C(A)$ is finite,

2) the separated quiver $\Gamma^{s}(A)$ of $A$ has no cycles (orientation ignored) and $\operatorname{dim}(e J(A)) \leq 3$ for every primitive idempotent $e \in A$.

One can easily give examples of non-basic algebras with a 2-nilpotent Jacobson radical which violate the criterion given above, in particular the bound on the dimension of $e J(A)$.

Example 4.3. Consider $A=M_{n}\left(K[x] /\left(x^{2}\right)\right)$.

It is clear that $A=A^{\prime} \oplus J(A)$ as vector spaces, where $A^{\prime}=M_{n}(K)$, $J(A)=M_{n}(K \bar{x})$, with $\bar{x}$ denoting the image of $x$ in $K[x] /\left(x^{2}\right)$, and $J(A)^{2}=0$. By $e_{i j}, i, j=1, \ldots, n$, we denote the matrix units in $A^{\prime}$. Let $L \in L(A)$. Let $L_{i}$ denote the $i$-th row of $L$, defined as the linear space $e_{i i} L$. Clearly $L=L_{1}+\cdots+L_{n}$.

Since $e_{i j} L \subseteq L$ for every $i, j$, all rows of $L$ are equal, as $K$-subspaces of $K^{n}$. Let $d(L)$ stand for the linear dimension of $L_{1}$. Assume that $L \subseteq J(A)$. Every invertible element of $A$ is of the form $u=u^{\prime}+r$, where $u^{\prime} \in U\left(A^{\prime}\right)=G L_{n}(K)$ and $r \in J(A)$. It follows that $L u=$ $L u^{\prime}$. If $M, N$ are subspaces of the space of row vectors $K^{n}$ of equal dimension, then there exists an element $u \in G L_{n}(K)$ such that $M u=N$. Consequently, for every $L, L^{\prime}$ contained in $J(A)$ the following equivalence holds:

$$
[L]=\left[L^{\prime}\right] \in C(A) \Longleftrightarrow d(L)=d\left(L^{\prime}\right) .
$$


It follows that $M_{n}\left(K[x] /\left(x^{2}\right)\right)$ has exactly $n+1$ conjugacy classes of left nilpotent ideals. Thus, by Theorem 1.1, the semigroup $C\left(M_{n}\left(K[x] /\left(x^{2}\right)\right)\right)$ is finite.

The dimension of $e J(A)$ could be arbitrarily large in the previous example. Clearly, the lattice of ideals of $A$ is distributive. This shows that a direct generalization of Theorem 4.2 to the non-basic case is not possible.

Definition 4.4. Assume that $A$ is a $K$-algebra with a complete set $\left\{e_{1}, e_{2}, \ldots, e_{n}\right\}$ of primitive orthogonal idempotents. By a basic algebra associated to $A$ we mean the algebra

$$
A^{b}=e_{A} A e_{A}
$$

where $e_{A}=e_{j_{1}}+\cdots+e_{j_{t}}$, and $e_{j_{1}}, \ldots, e_{j_{t}}$ are chosen so that $A e_{j_{i}} \not A e_{j_{s}}$ for $i \neq s$ and each $A$-module $A e_{j}$ is isomorphic to one of the modules $A e_{j_{1}}, \ldots, A e_{j_{t}}$.

It is well known that $A^{b}$ does not depend on the choice of the sets $e_{1}, e_{2}, \ldots, e_{n}$ and $e_{j_{1}}, \ldots, e_{j_{t}}$, up to a $K$-algebra isomorphism (see [2, 6.5]). The algebra $A^{b}$ is basic and there is an equivalence of categories (of left modules) $\bmod A \cong \bmod A^{b}$.

We conclude with two observations concerning the relation between the finiteness of $C(A)$ and the finiteness of $C\left(A^{b}\right)$. We write $[L]_{e}$ for the conjugacy class of a left ideal $L$ in $e A e$, where $e=e^{2} \in A$.

Proposition 4.5. Assume that $e=e^{2}$ is an idempotent of a finite dimensional algebra $A$. Let $\phi\left([L]_{e}\right)=[A L]$ for $L \in L(e A e)$. Then $\phi: C(e A e) \rightarrow C(A)$ is well defined and it is a semigroup monomorphism. Proof: Assume that $\left[L_{1}\right]_{e}=\left[L_{2}\right]_{e}$ in $C(e A e)$. Then there exists $u \in$ $U(e A e)$ such that

$$
L_{1}=L_{2} u \text {. }
$$

Observe that $u+(1-e) \in U(A)$. Choose $v \in U(e A e)$ such that $u v=e$. Then

$$
(u+(1-e))(v+(1-e))=1 .
$$

Therefore (13) yields

$$
A L_{1}=A L_{2} u=A L_{2} e u=A L_{2} e(u+(1-e))=A L_{2}(u+1-e)
$$

and hence $\left[A L_{1}\right]=\left[A L_{2}\right]$ in $C(A)$. The map $\phi$ is therefore well defined. The multiplicativity of $\phi$ is clear. Indeed, for any $\left[L_{1}\right]_{e},\left[L_{2}\right]_{e} \in C(e A e)$ we have

$$
\begin{aligned}
\phi\left(\left[L_{1}\right]_{e}\left[L_{2}\right]_{e}\right) & =\phi\left(\left[L_{1} L_{2}\right]_{e}\right)=\left[A L_{1} L_{2}\right]=\left[A L_{1} e A e L_{2}\right] \\
& =\left[A L_{1} A L_{2}\right]=\left[A L_{1}\right]\left[A L_{2}\right]=\phi\left(\left[L_{1}\right]_{e}\right) \phi\left(\left[L_{2}\right]_{e}\right) .
\end{aligned}
$$


If $\left[A L_{1}\right]=\left[A L_{2}\right]$ then $A L_{1}=A L_{2} g$ for some $g \in U(A)$. Hence $L_{1}=$ $e A L_{1}=e A L_{2} g=L_{2} g$, so that $L_{1}=L_{2}(e g e)$. A similar argument shows that $L_{2}=L_{1}(e h e)$ for some $h \in U(A)$. Let $g^{\prime}=e g e, h^{\prime}=e h e$. Proceeding as in the proof of Proposition 3.3 we find maximal subgroups $H, H^{\prime}$ of the multiplicative monoid $e A e$ such that $\left(h^{\prime} g^{\prime}\right)^{n} \in H,\left(g^{\prime} h^{\prime}\right)^{n} \in H^{\prime}$ for some $n \geq 1$. As before, it follows that $\left(h^{\prime} g^{\prime}\right)^{n},\left(g^{\prime} h^{\prime}\right)^{n}$ generate the same ideal of this monoid and

$$
L_{1}=L_{1} h^{\prime} g^{\prime}=L_{1}\left(h^{\prime} g^{\prime}\right)^{n}, \quad L_{2}=L_{2} g^{\prime} h^{\prime}=L_{2}\left(g^{\prime} h^{\prime}\right)^{n} .
$$

Let $f, f^{\prime}$ stand for the unit elements of $H, H^{\prime}$ respectively. Then, similarly to (10) we have

$$
L_{1}=L_{1} f, \quad L_{2}=L_{2} f^{\prime}
$$

and by Proposition 2.2 the idempotents $f \in H, f^{\prime} \in H^{\prime}$ are conjugate in $e A e$. Replacing $L_{2}$ by its conjugate in $e A e$ we may assume that $f=f^{\prime}$ and thus $H=H^{\prime}$. Therefore $\left(h^{\prime} g^{\prime}\right)^{n} h^{\prime},\left(g^{\prime} h^{\prime}\right)^{n} g^{\prime} \in H=U(f A f)$. Consider $z=e-f+\left(h^{\prime} g^{\prime}\right)^{n} h^{\prime}$. Then $z \in U(e A e)$ and clearly $f(e-f)=0$. From (14) and (15) we deduce that

$$
L_{1} z=L_{1} f z=L_{1}\left(h^{\prime} g^{\prime}\right)^{n} h^{\prime}=L_{1} h^{\prime}=L_{2} .
$$

Therefore the classes $\left[L_{1}\right]_{e}$ and $\left[L_{2}\right]_{e}$ are equal in $C(e A e)$. The injectivity of $\phi$ follows.

Corollary 4.6. If $C(A)$ is finite then $C\left(A^{b}\right)$ is finite.

Note that the finiteness of $C\left(A^{b}\right)$ does not imply the finiteness of $C(A)$. Indeed, suppose that if $C(B)$ is finite for some algebra $B$ then every $C\left(M_{n}(B)\right)$ is finite, for $n \geq 1$. From Theorem 7 in [8] it follows that $B$ is of finite representation type. However, one can easily give an example of an algebra $B$ that is of infinite representation type and has finitely many conjugacy classes of left ideals.

Example 4.7. Consider the following subalgebra $B$ of the matrix algebra $M_{7}(K)$

$$
\left(\begin{array}{ccccccc}
K & K & K & 0 & K & 0 & 0 \\
0 & K & 0 & 0 & 0 & 0 & 0 \\
0 & 0 & K & 0 & 0 & 0 & 0 \\
0 & 0 & 0 & K & K & K & K \\
0 & 0 & 0 & 0 & K & 0 & 0 \\
0 & 0 & 0 & 0 & 0 & K & 0 \\
0 & 0 & 0 & 0 & 0 & 0 & K
\end{array}\right) .
$$

Then algebra $B$ is of infinite representation type. However, $C(B)$ is finite. 
Proof: Clearly, $B$ is a basic algebra and $J(B)^{2}=0$. Moreover $\operatorname{dim}(e J(B)) \leq 3$ for every primitive idempotent $e$ of $B$. It is easy to see that the separated graph $\Gamma^{s}(B)$ is of the following form

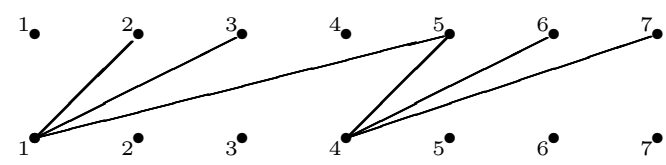

Hence, $\Gamma^{s}(B)$ has no cycles. From Theorem 4.2 it follows that the semigroup $C(B)$ is finite. However, the separated graph $\Gamma^{s}(B)$ is not a disjoint union of Dynkin graphs. Thus, according to Theorem 11.8 of [9], the algebra $B$ is not of finite representation type.

\section{Some recognizable objects}

Since $A$ is a finite dimensional algebra over an algebraically closed field $K$, we may write

$$
A / J(A) \cong M_{s_{1}}(K) \oplus M_{s_{2}}(K) \oplus \cdots \oplus M_{s_{n}}(K),
$$

for some positive integers $s_{i}, i=1,2, \ldots, n$. By the classical WedderburnMalcev theorem $\left[2\right.$, I.1.6] there exists a subalgebra $A^{\prime}$ of $A$ such that $A=$ $A^{\prime} \oplus J(A)$ as subspaces and the restriction of $\pi: A \rightarrow A / J(A)$ to $A^{\prime}$ is an isomorphism of algebras. Then $A^{\prime}$ is a direct product of simple algebras $A_{i}$, for $1 \leq i \leq n$, and by (16) we may assume that $A_{i} \cong M_{s_{i}}(K)$. Hence, we get the following decomposition of the $K$-space $A$

$$
A=A^{\prime} \oplus J(A)=A_{1} \oplus A_{2} \oplus A_{3} \oplus \cdots \cdots \oplus A_{n} \oplus J(A), \quad 1 \leq i \leq n .
$$

Recall that $\bar{x}$ denotes the image $\pi(x)$ of $x \in A$ in $A / J(A)$. We shall distinguish between the algebra $A_{i}$ and its isomorphic copy $\overline{A_{i}}=\pi\left(A_{i}\right)$.

Definition 5.1. By the radical of the semigroup $C(A)$ we mean the largest semigroup ideal of $C(A)$ consisting of nilpotent elements. The radical of $C(A)$ will be denoted by $\mathcal{N}(C(A))$.

Observe that we have the following equality

$$
\mathcal{N}(C(A))=\{[L] \in C(A) \mid L \subseteq J(A)\} .
$$

Therefore $\mathcal{N}(C(A))$ is the set of all nilpotent elements of $C(A)$ and it is the largest nilpotent ideal of $C(A)$.

Definition 5.2. We say that elements $[X],[Y] \in C(A)$ are orthogonal modulo $\mathcal{N}(C(A))$ if $[X Y] \in \mathcal{N}(C(A))$. A subset $\left\{\left[L_{1}\right], \ldots,\left[L_{m}\right]\right\}$ of 
$C(A) \backslash \mathcal{N}(C(A))$ is called a full orthogonal set modulo $\mathcal{N}(C(A))$ if it is a maximal subset of $C(A) \backslash \mathcal{N}(C(A))$, for which we have

$$
\left[L_{i}\right]\left[L_{j}\right] \in \mathcal{N}(C(A)), \text { for } 1 \leq i, j \leq m, i \neq j .
$$

We start with the following observation.

Remark 5.3. Let $\left\{\left[L_{1}\right], \ldots,\left[L_{m}\right]\right\}$ be a full orthogonal set modulo $\mathcal{N}(C(A))$. Then there do not exist $i, j \in\{1,2, \ldots, m\}$ and $k \in\{1,2, \ldots, n\}$, such that $i \neq j$ and

$$
\overline{L_{i}} \cap \overline{A_{k}} \neq 0, \quad \overline{L_{j}} \cap \overline{A_{k}} \neq 0 .
$$

Proof: Suppose on the contrary that there exist $i \neq j \in\{1,2, \ldots, m\}$ and $k \in\{1,2, \ldots, n\}$ such that $(18)$ holds. Then $\left(\overline{L_{i}} \cap \overline{A_{k}}\right)\left(\overline{L_{j}} \cap \overline{A_{k}}\right) \neq 0$, whence $\left[L_{i} L_{j}\right] \notin \mathcal{N}(C(A))$. This contradicts the hypothesis.

Proposition 5.4. The following conditions are equivalent for any collection $L_{1}, L_{2}, \ldots, L_{m}$ of left ideals of $A$ :

(i) $\left\{\left[L_{1}\right], \ldots,\left[L_{m}\right]\right\}$ is a full orthogonal set modulo $\mathcal{N}(C(A))$,

(ii) $m=n$, the number of simple blocks in $A / J(A)$ as in (17), and there exists a permutation $\sigma \in S_{n}$ such that $0 \neq \overline{L_{\sigma(i)}} \subseteq \overline{A_{i}}$.

Proof: Assume that (i) holds. By Remark 5.3 the cardinality of any full orthogonal set modulo $\mathcal{N}(C(A))$ does not exceed the number of simple blocks in $A / J(A)$. Suppose that $m<n$. By Remark 5.3 there exists $i \in\{1,2, \ldots, n\}$ such that

$$
\overline{L_{j}} \cap \overline{A_{i}}=0, \quad \text { for all } 1 \leq j \leq m .
$$

Thus $\left(\overline{A_{i}+J(A)}\right) \overline{L_{j}}=\overline{A_{i} L_{j}}=0$, for every $1 \leq j \leq m$. Hence, all pairs: $\left[A_{i}+J(A)\right],\left[L_{j}\right]$ are, for $1 \leq j \leq m$, orthogonal modulo $\mathcal{N}(C(A))$. This contradicts (i), proving that $m=n$. It is clear that there exists a permutation $\sigma \in S_{n}$ such that $\overline{L_{\sigma(i)}} \subseteq \overline{A_{i}}$.

Assume that (ii) holds. Clearly, the elements of the set $\left\{\left[L_{1}\right], \ldots,\left[L_{n}\right]\right\}$ are pairwise orthogonal modulo $\mathcal{N}(C(A))$. If this is not a full orthogonal set then there exists $[L] \in C(A) \backslash \mathcal{N}(C(A))$, orthogonal modulo $\mathcal{N}(C(A))$ to every $\left[L_{j}\right]$, where $1 \leq j \leq n$. Therefore, there exists $k \in\{1,2, \ldots, n\}$ such that $\bar{L} \cap \overline{A_{k}} \neq 0$. Hence $\overline{L L_{\sigma(k)}} \neq 0$, which contradicts the fact that $[L]$ and $\left[L_{k}\right]$ orthogonal modulo $\mathcal{N}(C(A))$. Thus (i) follows.

Example 5.5. The elements $\left[L_{i}\right], 1 \leq i \leq n$, defined by

$$
L_{i}=\pi^{-1}\left(\overline{A_{i}}\right)=A_{i}+J(A)
$$

form a full orthogonal set modulo $\mathcal{N}(C(A))$. 
We fix a full orthogonal set modulo $\mathcal{N}(C(A))$, say $\mathcal{L}=\left\{\left[L_{1}\right], \ldots,\left[L_{n}\right]\right\}$. We may assume that $\overline{L_{i}} \subseteq \overline{A_{i}}$, for $1 \leq i \leq n$, according to Proposition 5.4. For every $i \in\{1,2, \ldots, n\}$ we define a subset $\mathcal{C}_{i}$ of $C(A)$ as the collection of all classes $[L] \in C(A)$ that satisfy the following conditions:

$$
\left\{\begin{array}{l}
{[L]\left[L_{i}\right] \notin \mathcal{N}(C(A)),} \\
{[L]\left[L_{j}\right] \in \mathcal{N}(C(A)), \quad j \neq i .}
\end{array}\right.
$$

It is clear that for $1 \leq i \leq n$ we have

$$
\mathcal{C}_{i}=\left\{[L] \in C(A) \mid 0 \neq \bar{L} \subseteq \overline{A_{i}}\right\} .
$$

Corollary 5.6. The description of the sets $\mathcal{C}_{i}$ given in (20) does not depend (up to the order) on the choice of a full orthogonal set modulo $\mathcal{N}(C(A))$.

We introduce the following subsets of $C(A)$

$$
\begin{aligned}
& \mathcal{J}_{i}=\left\{[L] \in \mathcal{N}(C(A)) \mid \mathcal{C}_{j} \cdot[L]=0, j \neq i\right\}, \\
& \mathcal{I}_{i}=\left\{[L] \in \mathcal{N}(C(A)) \mid[L] \cdot \mathcal{C}_{j}=0, j \neq i\right\} .
\end{aligned}
$$

Clearly, the radical $\mathcal{N}(C(A))$, the collection $\mathcal{C}_{i}, i=1, \ldots, n$, and the sets $\mathcal{J}_{i}, \mathcal{I}_{i}$ are recognizable in terms of the semigroup $C(A)$.

\section{Is $A$ determined by $C(A)$ ?}

Our main aim in this section is to prove Theorem 1.2. Therefore, in what follows we assume that $J(A)^{2}=0$. Let $J_{i j}=A_{i} J(A) A_{j}$. Then $J_{i j}$ are subspaces of $J(A)$ and in the matrix notation we can write

$$
A=A^{\prime} \oplus J(A)=\left(\begin{array}{cccc}
A_{1} & 0 & \ldots & 0 \\
0 & A_{2} & \ldots & 0 \\
\vdots & \vdots & \ddots & \vdots \\
0 & 0 & \ldots & A_{n}
\end{array}\right) \oplus\left(\begin{array}{cccc}
J_{11} & J_{12} & \ldots & J_{1 n} \\
J_{21} & J_{22} & \ldots & J_{2 n} \\
\vdots & \vdots & \ddots & \vdots \\
J_{n 1} & J_{n 2} & \ldots & J_{n n}
\end{array}\right)
$$

According to (17) simple algebras $A_{i}$ are isomorphic to $M_{s_{i}}(K)$, for $1 \leq i \leq n$. Then $J_{i j}$ are $A_{i}-A_{j}$-bimodules. Hence $J_{i j}$ are right modules over the algebra $A_{i}^{o p} \otimes_{K} A_{j} \cong M_{s_{i} s_{j}}(K)$. So they are of the form

$$
J_{i j}=\underbrace{N_{i j} \oplus N_{i j} \oplus \cdots \oplus N_{i j}}_{m_{i j}},
$$

where $m_{i j} \in \mathbb{N}$, and $N_{i j}$ is isomorphic to a minimal right ideal of $M_{s_{i} s_{j}}(K)$.

We will show that if $A$ satisfies the hypotheses of Theorem 1.2, then the properties of the semigroup $C(A)$ allow us to determine the set $\left\{s_{1}, s_{2}, \ldots, s_{n}\right\}$, as well as the matrix $\left[m_{i j}\right]$. 
Proposition 6.1. Assume that $A$ is a finite dimensional algebra over an algebraically closed field $K$ such that $J(A)^{2}=0$ and $C(A)$ is a finite semigroup. Then the lattice of ideals of $A$ is distributive. Moreover, if

$$
\mathcal{J}_{i j}=\mathcal{J}_{i} \cap \mathcal{I}_{j}, \text { for } 1 \leq i, j \leq n,
$$

and $\left[m_{i j}\right]$ is the matrix corresponding to the algebra $A$, as in (24), then

$$
m_{i j}=\left\{\begin{array}{ll}
0 & \text { if } \mathcal{J}_{i j}=\{[0]\} \\
1 & \text { if } \mathcal{J}_{i j} \neq\{[0]\}
\end{array} .\right.
$$

Proof: From Theorem 6 in [8] we know that the lattice of ideals of $A$ is distributive. From Corollary 2.4c in [9] it follows that all entries of the matrix $\left[m_{i j}\right]$ are in the set $\{0,1\}$. As an easy consequence of (22) and (23) we get

$$
\mathcal{J}_{i j}=\left\{[L] \in C(A) \mid L \subseteq J_{i j}\right\} .
$$

The assertion follows from (24).

Corollary 6.2. If $A$ is as in Proposition 6.1 and $\mathcal{J}_{i j} \neq 0$ for some $i, j$, then the nonzero elements of $\mathcal{J}_{i j}$ form a $\mathcal{J}$-class in $C(A)$. The cardinality of this $\mathcal{J}$-class is equal to $s_{j}$.

Proof: Since $m_{i j}=1$ by (25), we know that $0 \neq J_{i j}$ is a simple right $M_{s_{i} s_{j}}(K)$-module, hence a simple right $A_{i}^{\mathrm{op}} \otimes A_{j}$-module. Thus, we can identify this module with the space of rectangular matrices $M_{s_{i} \times s_{j}}(K)$ with the natural actions of $A_{i}$ and $A_{j}$.

Let $L$ be a left ideal of $A$ contained in $J_{i j}$. Then

$$
L=f_{1,1} L \oplus \cdots \oplus f_{s_{i}, s_{i}} L,
$$

where $f_{i, i}$ are, for $1 \leq i \leq s_{i}$, primitive diagonal idempotents in $A_{i}$. As in Example 4.3, we get that $f_{1,1} L, \ldots, f_{s_{i}, s_{i}} L$ are isomorphic as subspaces of $K^{s_{j}}$. In fact, if $f_{k, m}$ are, for $1 \leq k, m \leq s_{i}$, the matrix units in $A_{j}$, then $f_{k, m} f_{m, m} L=f_{k, k} L$ and $f_{m, m} L \cong f_{k, k} L$.

If $L^{\prime} \subseteq J_{i j}$ is such that $\operatorname{dim}(L)=\operatorname{dim}\left(L^{\prime}\right)$ then $\operatorname{dim}\left(f_{1,1} L\right)=\operatorname{dim}\left(f_{1,1} L^{\prime}\right)$ and $f_{1,1} L, f_{1,1} L^{\prime} \subseteq K^{s_{j}}$. Hence, there exists $a \in U\left(A_{j}\right)$ such that $f_{1,1} L=$ $f_{1,1} L^{\prime} a$. Then it is easy to see that $L=L^{\prime} a$. Similarly one gets $L^{\prime}=L b$, for some $b \in U\left(A_{j}\right)$. As in the proof of Theorem 1.1, it follows that $\left[L^{\prime}\right]=[L]$. Hence, from $(26)$ we get

$$
\left|\mathcal{J}_{i j}\right| \leq s_{j}+1 .
$$

We know that $J_{i j}=M_{1} \oplus \cdots \oplus M_{s_{j}}$, where $M_{i}$ are left ideals in $A$, and each of them is a simple left $A_{i}$-module. Clearly, the elements

$$
\left[M_{1} \oplus \cdots \oplus M_{k}\right], \quad k=1, \ldots, s_{j}
$$


are different because $M_{1} \oplus \cdots \oplus M_{k}$ have different dimensions as $K$-spaces. We also know that

$$
\left(M_{1} \oplus \cdots \oplus M_{k}\right)\left(A A_{j}\right)=J_{i j},
$$

because $J_{i j}$ is a simple bimodule. Also

$$
J_{i j}(A f)=\left(M_{1} \oplus \cdots \oplus M_{k}\right)
$$

for a diagonal idempotent $f$ of rank $k$ in $A_{j} \cong M_{s_{j}}(K)$. All elements $\left[M_{1} \oplus \cdots \oplus M_{k}\right]$ are therefore in the same $\mathcal{J}$-class of the semigroup $C(A)$. Thus, the assertion follows from (27).

Let $e \in A_{j}$ be an idempotent. Then the conjugacy class of $A_{j} e$ in $A_{j}$ (that is, an element of $C\left(A_{j}\right)$ ) is denoted by $\left[A_{j} e\right]_{A_{j}}$.

Lemma 6.3. Assume that $j$ is such that $\mathcal{J}_{i j}=0$ for every $i \in\{1,2, \ldots, n\}$. Let $e_{1}, e_{2}$ be idempotents in $A_{j}$. Then

$$
\left[A e_{1}\right]=\left[A e_{2}\right] \text { in } C(A) \Longleftrightarrow\left[A_{j} e_{1}\right]_{A_{j}}=\left[A_{j} e_{2}\right]_{A_{j}} \text { in } C\left(A_{j}\right) \text {. }
$$

Proof: Since $\mathcal{J}_{i j}=0$ for $i=1,2, \ldots, n$, we get $J(A) A_{j}=0$ and $A_{j}$ is a left ideal of $A$. Therefore $\left[A e_{1}\right]=\left[A_{j} e_{1}\right]$, for every idempotent $e_{1} \in$ $A_{j}$. The proof of (28) is therefore reduced to the proof of the following equivalence

$$
\left[A_{j} e_{1}\right]=\left[A_{j} e_{2}\right] \text { in } C(A) \Longleftrightarrow\left[A_{j} e_{1}\right]_{A_{j}}=\left[A_{j} e_{2}\right]_{A_{j}} \text { in } C\left(A_{j}\right) .
$$

Let $g \in U(A)$ be such that $A_{j} e_{1}=A_{j} e_{2} g$. From (17) we get a decomposition $g=g_{1}+g_{2}+\cdots+g_{n}+z$, where $g_{i} \in U\left(A_{i}\right)$ and $z \in J(A)$. Then

$$
A_{j} \supseteq A_{j} e_{1}=A_{j} e_{2} g=A_{j} e_{2}\left(g_{j}+z\right) .
$$

Hence $A_{j} e_{1}=A_{j} e_{2} g_{j}$, and so $\left[A_{j} e_{1}\right]_{A_{j}}=\left[A_{j} e_{2}\right]_{A_{j}}$ in $C\left(A_{j}\right)$.

Conversely, assume that $\left[A_{j} e_{1}\right]_{A_{j}}=\left[A_{j} e_{2}\right]_{A_{j}}$ in $C\left(A_{j}\right)$, so that $A_{j} e_{1}=$ $A_{j} e_{2} u$, for some $u \in U\left(A_{j}\right)$. Let $E_{j}$ be the unity of the algebra $A_{j}$. Then $u+\left(1-E_{j}\right) \in U(A)$. Moreover, $A_{j} e_{2}\left(u+\left(1-E_{j}\right)\right)=A_{j} e_{2} u=A_{j} e_{1}$. Therefore $\left[A_{j} e_{1}\right]=\left[A_{j} e_{2}\right]$. We have proved that $(29)$ holds, hence also proving (28).

Corollary 6.4. Assume that $j$ is such that $\mathcal{J}_{i j}=0$ for every $i \in$ $\{1,2, \ldots, n\}$. Then

$$
\left|\mathcal{C}_{j}\right|=s_{j} \cdot|\mathcal{N}(C(A))| .
$$

Proof: Let $[L] \in C_{j}$. Then

$$
L=A e \oplus L(1-e),
$$

for some maximal idempotent $e$ in $L$ and $L(1-e) \subseteq J(A)$ (see Lemma 3.1). Moreover, by (21) and Proposition 2.2, $[L]=\left[L^{\prime}\right]$ for $L^{\prime}=g^{-1} L g$, where 
$g \in U(A)$ is such that $e^{\prime}=g^{-1} e g \in A_{j}$. Hence, replacing $L$ by $L^{\prime}$, we may assume that $e \in A_{j}$.

On the other hand, if we fix some $e=e^{2} \in A_{j}$, then for every $L_{0}<_{l} A$ such that $L_{0} \subseteq J(A)$ we have $L_{0}=L_{0}(1-e)$ because $J(A) A_{j}=0$. Then $L=A e \oplus L_{0}=A_{j} e \oplus L_{0}$ is a left ideal in $A$. Moreover, $e$ is a maximal idempotent in $L$, by Lemma 3.1, and clearly $L_{0}=J(L)$. If $\left[L^{\prime}\right] \in C_{j}$ then we may write $L^{\prime}=A e^{\prime} \oplus L_{0}^{\prime}$, where $e^{\prime}=\left(e^{\prime}\right)^{2} \in A_{j}$ and $L_{0}^{\prime} \subseteq J(A)$. If $[L]=\left[L^{\prime}\right]$ then $e, e^{\prime}$ must be conjugate by Proposition 2.2, so that $[A e]=\left[A e^{\prime}\right]$. Since $L^{\prime}=u^{-1} L u$ for some $u \in U(A)$, we also get $L_{0}^{\prime}=J\left(L^{\prime}\right)=u^{-1} J(L) u=u^{-1} L_{0} u$, so that $\left[L_{0}\right]=[L]$. In view of Lemma 6.3, the assertion follows from Proposition 3.3 and from the fact that $C\left(A_{j}\right)$ has $s_{j}$ nonzero elements, each of the form $\left[A_{j} e\right]_{A_{j}}$, for $e=e^{2} \in A_{j}$.

Proof of Theorem 1.2: According to (17) and (24), an algebra $A$ such that $C(A)$ finite and $J(A)^{2}=0$ determines a pair $\left(\nu_{A}, \mu_{A}\right)$ consisting of a nondecreasing sequence of natural numbers $\nu_{A}=\left(s_{1}, s_{2}, \ldots, s_{n}\right)$ (this can be assumed after a permutation of the blocks $\left.A_{1}, A_{2}, \ldots, A_{n}\right)$ and a matrix $\mu_{A}=\left[m_{i j}\right]$ in $M_{n}\left(\mathbb{Z}_{2}\right)$.

By $[\mathbf{9}$, Exercise $1, \S 11.8]$, for every pair $(\nu, \mu)$ as above there exists a $K$-algebra $A(\nu, \mu)=N \rtimes A^{\prime}$, where $A^{\prime}=A_{1} \oplus A_{2} \oplus \cdots \oplus A_{n}$, with $a_{i} \cong M_{s_{i}}(K)$, and $N$ is a $A^{\prime}$-bimodule

$$
\bigoplus_{1 \leq i, j \leq n} \bigoplus m_{i j} N_{i j}
$$

where $N_{i j}$ are minimal right ideals in $A_{i}^{o p} \otimes A_{j}$ and $N^{2}=0$. Moreover, every finite dimensional $K$-algebra $A$ such that $J(A)^{2}=0$ is isomorphic to the algebra of the form $A\left(\nu_{A}, \mu_{A}\right)$.

Let $\nu=\left(s_{1}, s_{2}, \ldots, s_{n}\right)$ be a nondecreasing sequence of natural numbers. By $G_{\nu}$ we denote the subgroup of the permutation group $S_{n}$ defined by

$$
G_{\nu}=\left\{\pi \in S_{n} \mid(\pi(i)=j) \quad \Rightarrow \quad\left(s_{i}=s_{j}\right)\right\} .
$$

Let $\mu=\left[m_{i j}\right]$ and $\mu^{\prime}=\left[m_{i j}^{\prime}\right]$ be matrices in $M_{n}(\mathbb{N})$. We define a relation $\sim_{\nu}$ by $\left[m_{i j}\right] \sim_{\nu}\left[m_{i j}^{\prime}\right]$ if and only if there exists $\pi \in G_{\nu}$ such that

$$
m_{i j}^{\prime}=m_{\pi(i) \pi(j)}, \text { for all } i, j .
$$

By [9, Exercise 1c, $\S 11.8]$, we know that $A(\nu, \mu) \cong A\left(\nu^{\prime}, \mu^{\prime}\right)$ if and only if $\nu=\nu^{\prime}$ and $\mu \sim_{\nu} \mu^{\prime}$.

Consider an algebra $B$ such that that the semigroups $C(A)$ and $C(B)$ are isomorphic. It is then clear that

$$
J(A)^{2}=0 \Longleftrightarrow \mathcal{N}(C(A))^{2}=0 \Longleftrightarrow \mathcal{N}(C(B))^{2}=0 \Longleftrightarrow J(B)^{2}=0 .
$$


Since $C(A)$ is finite, $C(B)$ also is finite and hence the lattice of ideals of $B$ is distributive. By Corollary 6.2 and Corollary 6.4 the fact that $C(A)$ and $C(B)$ are isomorphic implies that the nondecreasing sequences describing the sizes of simple blocks of the algebras $A / J(A)$ and $B / J(B)$, namely the sequences $\nu_{A}=\left(s_{1}, s_{2}, \ldots, s_{n}\right)$ and $\nu_{B}=\left(s_{1}^{\prime}, s_{2}^{\prime}, \ldots, s_{n}^{\prime}\right)$, are equal. Since $C(A) \cong C(B)$, from Proposition 6.1 it follows also easily that $\mu_{A} \sim_{\nu} \mu_{B}$. Therefore, by [9, Exercise 1c, §11.8], we know that $A\left(\nu_{A}, \mu_{A}\right) \cong A\left(\nu_{B}, \mu_{B}\right)$, and hence also $A \cong B$.

\section{References}

[1] F. W. Anderson And K. R. Fuller, "Rings and categories of modules", Second edition, Graduate Texts in Mathematics 13, Springer-Verlag, New York, 1992. DOI : 10.1007/978-1-4612-4418-9.

[2] I. Assem, D. Simson, And A. Skowroński, "Elements of the representation theory of associative algebras. Vol. 1. Techniques of representation theory", London Mathematical Society Student Texts 65, Cambridge University Press, Cambridge, 2006. DOI: 10.1017/СB09780511614309.

[3] A. H. Clifford and G. B. Preston, "The algebraic theory of semigroups", Vol. I, Mathematical Surveys 7, American Mathematical Society, Providence, R.I., 1961.

[4] Y. A. Drozd And V. V. Kirichenko, "Finite dimensional algebras", Translated from the 1980 Russian original and with an appendix by Vlastimil Dlab, Springer-Verlag, Berlin, 1994. DOI: 10.1007/978-3-642-76244-4.

[5] Y. Hirano, Rings with finitely many orbits under the regular action, in: "Rings, modules, algebras, and abelian groups", Lecture Notes in Pure and Appl. Math. 236, Dekker, New York, 2004, pp. 343-347.

[6] E. Jespers and J. Okniński, Descending chain conditions and graded rings, J. Algebra 178(2) (1995), 458-479. DOI: 10.1006/ jabr.1995.1360.

[7] T. Y. LAM, "A first course in noncommutative rings", Second edition, Graduate Texts in Mathematics 131, Springer-Verlag, New York, 2001. DOI : 10.1007/978-1-4419-8616-0.

[8] J. OKniński And L. E. Renner, Algebras with finitely many orbits, J. Algebra 264(2) (2003), 479-495. DOI: 10.1016/S00218693(03) 00129-7. 
[9] R. S. Pierce, "Associative algebras", Graduate Texts in Mathematics 88, Studies in the History of Modern Science 9, SpringerVerlag, New York-Berlin, 1982.

[10] M. S. PutchA, "Linear algebraic monoids", London Mathematical Society Lecture Note Series 133, Cambridge University Press, Cambridge, 1988. DOI : $10.1017 /$ СB09780511600661.

Institute of Mathematics

University of Warsaw

Banacha 2

02-097 Warsaw

Poland

E-mail address: am234204@students.mimuw.edu.pl

E-mail address: okninski@mimuw.edu.pl

Primera versió rebuda el 5 de juny de 2012, darrera versió rebuda el 13 de setembre de 2012 . 